\title{
Highlights from the latest articles in nanomedicine for reproductive oncology
}

\section{Mesoporous silica nanoparticles loaded with peptide used as a biosensor to monitor the activity of anticancer drugs \\ Evaluation of: Townley HE, Zheng Y, Goldsmith J et al. A novel biosensor for quantitative monitoring of on-target activity of paclitaxel. Nanoscale 7(3), 1127-1135 (2015).}

Paclitaxel is a well-characterized potent antimitotic drug applied for the treatment of malignancies, including ovarian and breast cancer, which are among the most common types of reproductive malignancies in women. Paclitaxel acts via the binding with $\beta$-tubulin, resulting in the stabilization of microtubules, which, due to an increased life, become suitable for subsequent detyrosination at the $\alpha$-tubulin loci by a specific enzyme carboxypeptidase A [1]. Paclitaxel belongs to the taxane family of anticancer chemotherapeutics, and is characterized by poor aqueous solubility, which limits its clinical use. The challenges of poor paclitaxel solubility, however, can be reversed via the use of nanomaterials. The main body of research into the use of nanoscale tools for reproductive oncology has been predominantly focused upon the development of hollow or porous nanoparticles, which can act as drug delivery systems, or composite chemical nanosensors for the detection of malignant lesions [2].

In a recent publication, Townley et al. reported promising results associated with the use of paclitaxel-loaded mesoporous silica nanoparticles (paclitaxel-MSNPs) with additional paclitaxel activity quantification capacity in an ovarian cancer cell line (SKOv3). The authors demonstrated mitotic arrest in SKOv3 cells following exposure to paclitaxel-MSNPs, and, therefore, the efficacy of NPs for intracellular delivery of paclitaxel. In addition, Townley et al. conjugated MSNPs with a fluorescent peptide to use as a biomarker for the paclitaxel-induced cleavage of magnetic nanocapsule in order to monitor enzymatic activity. Furthermore, a fluorescent resonance energy transfer (FRET)-based peptide, which allows for the dualization visualization of fluorescent signal after peptide cleavage, was synthesized and used as an independent reporter molecule. Similar encouraging results were obtained as with the MSNP-conjugated peptide, suggesting that a FRET-based assay could be used to measure endogenous carboxypeptidase activity. In summary, the authors presented a novel combination of NP-based paclitaxel preparation and a FRET-based peptide biosensor for the measurement of on-target activity of paclitaxel. These exciting proof-ofconcept experiments demonstrate the validity of FRET methods for determining the efficacy of nanoscale drug delivery systems, and provide additional supporting evidence for the use of nanomaterials to improve the performance of existing chemotherapeutic drugs.

\section{Targeted biocompatible gold} nanoparticles for the delivery of platinum-based anticancer drugs

Evaluation of: Kumar A, Huo S, Zhang $X$ et al. Neuropilin-1-targeted gold nanoparticles enhance therapeutic efficacity of platinum (IV) drug for prostate cancer treatment. ACS Nano 8(5), 4205-4220 (2014).

Platinum-based antineoplastic drugs (platins) have been applied as a powerful tool for can-
Celine Jones ${ }^{\ddagger}, 1$, Natalia ${\text { Barkalina*, }{ }^{*, 1} \text { \& Kevin Coward }}^{1}$ 'Nuffield Department of Obstetrics \& Gynaecology, University of Oxford, Level 3, Women's Centre, John Radcliffe Hospital, Headington, Oxford, OX3 9DU, UK

*Author for correspondence: natalia.barkalina@obs-gyn.ox.ac.uk ${ }^{\ddagger}$ Authors contributed equally 
cer treatment over recent decades [3]. Platins represent a highly potent family of anticancer chemotherapeutics, acting via the induction of DNA damage and subsequent apoptosis. However, the clinical use of platins is associated with two key issues: dose-limiting systemic side effects and high levels of drug resistance development in cancer cells. From this perspective, the use of biomedical nanotechnology for the targeted intracellular delivery of platins could be particularly beneficial since it would allow the reduction of systemic toxicity and, possibly, overcome drug resistance due to the activation of alternative uptake mechanisms.

In a recent paper, Kumar et al. describe a novel approach, which improves the efficacy of delivery and optimizes the profile of platinum (IV; $\mathrm{Pt}[\mathrm{IV}]$ ) side effects, based upon the use of specialized gold NPs. The authors have previously reported the ability of a specific 5-amino acid peptide (CRGDK) to bind the CendR motif of Neuropilin-1, a transmembrane glycoprotein abundantly expressed by a variety of malignant cell types and receptors, which promotes tissue penetration [4]. In this study, the authors synthesized small glutathione-stabilized gold NPs (Au@GSH) with inherent antioxidant properties associated with the reactive oxygen species-neutralizing capacity of glutathione, functionalized with $\mathrm{Pt}(\mathrm{IV})$ and CRGDK (Au@Pt[IV] + CRGDK). They assessed the cytotoxicity of this novel nanoplatform by comparing the effects of unconjugated NPs, free Pt(IV) and CRGDK peptide, and $\mathrm{Pt}(\mathrm{IV})$ and/or peptide-functionalized NPs in two different prostate cell lines (Nrp-1-positive PC-3 and Nrp-1-negative Du-145). Overall, the cytotoxicity and uptake of nanocarrier were increased in the presence of peptide (Au@Pt[IV] + CRGDK), compared with free Pt(IV) and Au@Pt(IV). At the same time, unconjugated NPs and free peptide were devoid of cytotoxicity. Collectively, the outcomes of this study further confirm that functionalization with targeting ligands enhances the drug delivery capacity of nanocarriers, and highlights the potential of targeted delivery vehicles to improve the tolerability of platinum drug-based therapies and reverse drug resistance.

\section{Biocompatible, biodegradable polymer nanoparticle delivery system for highly effective, low concentration of drugs} Evaluation of: Devulapally R, Sekar NM, Sekar TV et al. Polymer nanoparticles mediated codelivery of antimiR-10b and antimiR-21 for achieving triple negative breast cancer therapy. ACS Nano 9(3), 2290-2302 (2015).

Triple-negative breast cancer (TNBC) leads to high mortality and accounts for $15 \%$ of all breast cancers. Antisense miRNAs have been reported to suppress tumor growth, and therefore, are increasingly viewed upon as strong candidates for targeted molecular anticancer therapy. However, systemic delivery of miRNAs remains challenging due to rapid degradation by enzymes and inefficient intracellular uptake. Antisense-miR21 and antisense-miR10b have been previously reported to interfere with cell proliferation. However, an efficient delivery tool is required to promote their internalization into cells. Poly(lactic-co-glycolic acid) (PLGA) is a biocompatible, biodegradable polymer, widely used for NP synthesis, with the attractive ability to prevent degradation of loaded cargo and ensure progressive cargo release [5].

In a recent publication, Devulapally et al. describe the successful coloading of two antisense-miRNAs into PLGA- $b$-PEG NPs, synthesized using a double emulsion methodology with two different emulsifiers, and characterized by higher loading efficiency. The resulting NPs, coloaded with antisense-miRNAs, targeting miR21 and miR10b, which represent well-studied mediators of antiapoptosis and metastasis, respectively, and reduced the viability of an MDA-MB-231 breast cancer cell line (TNBC) in a dose-dependent fashion. In addition, coloading with two antisense-miRNAs potentiated the effects of treatment, compared with monotherapy with antisense-miR 21 or antisensemiR10b in TNBC cells and successfully suppressed tumor growth in tumor xenografts in mice in vivo. In an additional set of experiments, the authors showed that functionalization of NPs coloaded with antisensemiR21 and antisense-miR10b with a targeting peptide reduced the growth of tumor xenografts in vivo by the additional $40 \%$, compared with coloaded nonfunctionalized NPs. These encouraging findings suggest that this delivery system could be successfully used for other anticancer therapies in order to permit the increased efficacy of treatment with lower doses of drugs.

\section{Enhanced drug uptake and cytotoxicity associated with the use of a magnetic nanoparticle drug delivery system and applied magnetic fields}

Evaluation of: Elumalai R, Patil S, Maliyakkal N et al. Protamine-carboxymethyl cellulose magnetic nanocapsules for enhanced delivery of anticancer drugs against drug resistant cancers. Nanomedicine doi:10.1016/j. nano.2015.01.005 (2015) (Epub ahead of print). Current approaches to anticancer chemotherapy rely primarily upon the use of drugs with nonselective cytotoxicity, affecting both malignant and 'healthy' cells, and high risks of treatment-associated development of multidrug resistance. NP-based delivery 
systems can help to attenuate these issues, while also being specifically targeted toward cancer cells.

In a study by Elumalai et al., hollow nanocapsules, synthesized from two oppositely charged protamine and carboxymethyl cellulose polyelectrolytes using a layerby-layer technique, were modified with superparamagnetic iron oxide $\left(\mathrm{Fe}_{3} \mathrm{O}_{4}\right)$ NPs, resulting in the production of magnetic nanocapsules (MNCs). The authors applied these MNCs for the delivery of doxorubicin and evaluated composite cytotoxicity in vitro in a specially derived doxorubicin-resistant cervical cancer cell line (DR-HeLa cells), and in mouse models in vivo. In addition, the effects of an externally applied magnetic field upon the function of MNCs were investigated. Doxorubicin-loaded nanocapsules demonstrated antitumor effects in DR-HeLa cells, in contrast to free doxorubicin, as the result of improvement in drug uptake and intracellular retention. The external magnetic field enhanced MNC distribution within the in vivo model, as evidenced by a clear accumulation of doxorubicin

\section{References}

1 Ahmed AA, Wang X, Lu Z et al. Modulating microtubule stability enhances the cytotoxic response of cancer cells to Paclitaxel. Cancer Res. 71(17), 5806-5817 (2011).

2 Barkalina N, Charalambous C, Jones C, Coward K. Nanotechnology in reproductive medicine: emerging applications of nanomaterials. Nanomedicine 10(5), 921-938 (2014).

3 Chen X, Wu Y, Dong H, Zhang CY, Zhang Y. Platinumbased agents for individualized cancer treatment. Curr. Mol. Med. 13(10), 1603-1612 (2013). in the gastric region after the placement of magnets in proximity of the abdominal area. This interesting study demonstrates that MNCs improve doxorubicin uptake, and offer an additional benefit of control over biodistribution in the presence of external magnetic field. The outcomes of this work highlight the exciting perspectives for the development of more efficient delivery systems for chemotherapeutic drugs, which can minimize side effects that are currently associated with the treatment of drug-resistant tumors [6].

\section{Financial \& competing interests disclosure}

The authors have no relevant affiliations or financial involvement with any organization or entity with a financial interest in or financial conflict with the subject matter or materials discussed in the manuscript. This includes employment, consultancies, honoraria, stock ownership or options, expert testimony, grants or patents received or pending, or royalties.

No writing assistance was utilized in the production of this manuscript.

4 Kumar A, Ma H, Zhang X et al. Gold nanoparticles functionalized with therapeutic and targeted peptides for cancer treatment. Biomaterials 33(4), 1180-1189 (2012).

5 Gdowski A, Ranjan A, Mukerjee A, Vishwanatha J. Development of biodegradable nanocarriers loaded with a monoclonal antibody. Int. J. Mol. Sci. 16(2), 3990-3995 (2015).

6 Wu M, Zhang D, Zeng Y, Wu L, Liu X, Liu J. Nanocluster of superparamagnetic iron oxide nanoparticles coated with poly (dopamine) for magnetic field-targeting, highly sensitive MRI and photothermal cancer therapy. Nanotechnology 26(11), 115102 (2015). 